\title{
2-乙烯基硝基苯-8-羟基喹啉锌配合物的合成及紫外、苂光性质研究
}

\author{
霍延平* 戴立立黄宝华张焜方岩雄刘军 \\ (广东工业大学轻工化工学院应用化学系 广州 510006)
}

\begin{abstract}
摘要 设计合成了 3 种 8-羟基喹啉衍生物配体: (E)-2-[2-(2-硝基苯基)乙烯基]-8-羊基喹啉(4a)，(E)-2-[2-(3-硝基苯基)乙 烯基]-8-差基喹啉 $(\mathbf{4 b}),(E)$-2-[2-(4-硝基苯基)乙烯基]-8-羟基喹啉 $\left(\mathbf{4} \mathbf{c}\right.$ )及其相应的锌配合物 $\mathbf{5 a} \sim \mathbf{5}$, 产物经 ${ }^{1} \mathrm{H}$ NMR, IR, MS 和元素分析技术进行了结构表征. 通过紫外滴定模拟了金属锌与配体的配位过程，分别测定了它们固态和溶液状 态下的荧光性质: 光谱显示化合物 $\mathbf{5 a} \sim \mathbf{5}$ 固体荧光光谱的 $\lambda_{\text {max }}$ 分别是 $596,625,592 \mathrm{~nm}$, 在 DMF 溶液中的 $\lambda_{\text {max }}$ 分别是 $562,536,618 \mathrm{~nm}$. 荧光光谱显示硝基位置的改变可以调控 8-羟基喹啉锌配合物的发光性质.
\end{abstract}

关键词 8-差基喹啉; 硝基苯; 锌配合物; 苂光光谱

\section{Synthesis and Photophysics of Novel Zn Metal Complexes Based on 2-Substituted-8-hydroxyquinoline with Nitrobenzene Groups}

\author{
Huo, Yanping* Dai, Lili Huang, Baohua Zhang, Kun Fang, Yanxiong Liu, Jun \\ (Faculty of Light Industry and Chemical Engineering, Guangdong University of Technology, Guangzhou 510006)
}

\begin{abstract}
Three 8-hydroxyquinoline derivatives (E)-2-[2-(2-nitrophenyl)vinyl]-8-hydroxyquinoline (4a), (E)-2-[2-(3-nitrophenyl)vinyl]-8-hydroxyquinoline (4b), (E)-2-[2-(4-nitrophenyl)vinyl]-8-hydroxyquinoline (4c) and their zinc complexes $\mathbf{5 a} \sim 5 \mathbf{c}$ have been synthesized and identified by ${ }^{1} \mathrm{H}$ NMR, FFIR, MS techniques and elemental analyses. The aggregation behavior of $\mathrm{Zn}(\mathrm{OAc})_{2}$ and the ligands $\mathbf{4 a} \sim \mathbf{4} \mathbf{c}$ in solution was investigated by UV-vis. The luminescence properties of compounds $\mathbf{5 a} \sim 5 \mathbf{c}$ were investigated by fluorescence spectra in solid and DMF solution at room temperature. The results showed that the $\lambda_{\max }$ of compounds $\mathbf{5 a} \sim \mathbf{5 c}$ were 596, 625 and $592 \mathrm{~nm}$ in solid, and were 562, 536 and $618 \mathrm{~nm}$ in DMF solution. The comparative study of the luminescent properties of $\mathbf{5 a} \sim \mathbf{5} \mathbf{c}$ showed that the emission $\lambda_{\max }$ can be tuned by introducing nitro groups at different positions of benzene.
\end{abstract}

Keywords 8-hydroxyquinoline; nitrobenzene; zinc complex; fluorescence

有机电致发光器件(OLED)具有视角宽、功耗低、 响应速度快、工艺简单、能实现全色显示等优点，因此 在平板显示和固体光源领域具有广阔的应用前景 ${ }^{[1 \sim 3]}$. 特别是自从 1987 年 Tang 等 ${ }^{[4]}$ 首次报道了工作电压低、 发光亮度高的 OLED 以来, 其研究工作取得了更快速地 发展. 对有机电致发光来说, 红、绿、蓝三基色发光器 件已经进入了实用化阶段, 由这三基色混合而得的白光 器件目前已经比较成熟. 蓝光与黄光复合发光能够实现 白光发射是制备白光 OLED 的一个重要方法, 因此黄光 OLED 的研发成为当前的热点 ${ }^{[5 \sim 11]}$. 8-差基喹啉金属配 合物及其衍生物具有很好的热稳定性和发光亮度, 是优 良的电子传输材料和发光材料 ${ }^{[4]}$. 研究表明 ${ }^{[12 \sim 15]}$ : 8 -羟 基喹啉金属配合物的苂光来自于喹啉环的 $\pi-\pi *$ 电子迁
移，电子由基态跃迁到低激发态为 $\pi$ - $\pi$ *电子跃迁，8-羊全 基喹啉的最高占据分子轨道 HOMO 主要定域在酚羟基 环的氧原子, 而最低空轨道 LUMO 主要定域在喹啉环 中的吡啶环的 $\mathrm{N}$ 原子和 2 位碳原子上，通过对配体作适 当的化学修饰，改变 2-位上取代基团的吸电子或给电子 性质,可以改变喹啉配合物的 HOMO 与最低未占据分子 轨道 LUMO 之间的能隙, 引起配合物在激发态的系间 窝跃速度、内转化速度、发射衰减速度等性质的变化, 从 而改变它们的发光性质. 硝基化合物中硝基是强的吸电 子基团，它的存在有利于电子注入 LUMO 能级，从而大 大提高这类材料的电子迁移率, 同时它又是良好的生色 团, 可以延长 $\pi-\pi$ 共轭体系, 使紫外光谱红移且强度增 加. 基于以上思路, 为了获得优良的电子传输材料和黄

* E-mail: tigerhuo1974@yahoo.com.cn

Received July 5, 2011; revised November 6, 2011; accepted November 24, 2011.

Project supported by the National Natural Science Foundation of China (Nos. 20802010, 21172047).

国家自然科学基金(Nos. 20802010, 21172047)资助项目. 
光材料，本工作利用邻、间、对三种不同位置硝基取代 的苯甲醛和 2-甲基-8-羊基喹啉反应，对 8-着基喹啉环 上的 2 位进行结构修饰, 合成出硝基位置不同的 3 个配 体 ${ }^{[16]}$, 再与锌配位合成了 3 个新的金属锌配合物, 并研 究了邻、间、对硝基位置对金属锌配合物发光波长的影 响. 合成路线见 Scheme 1.

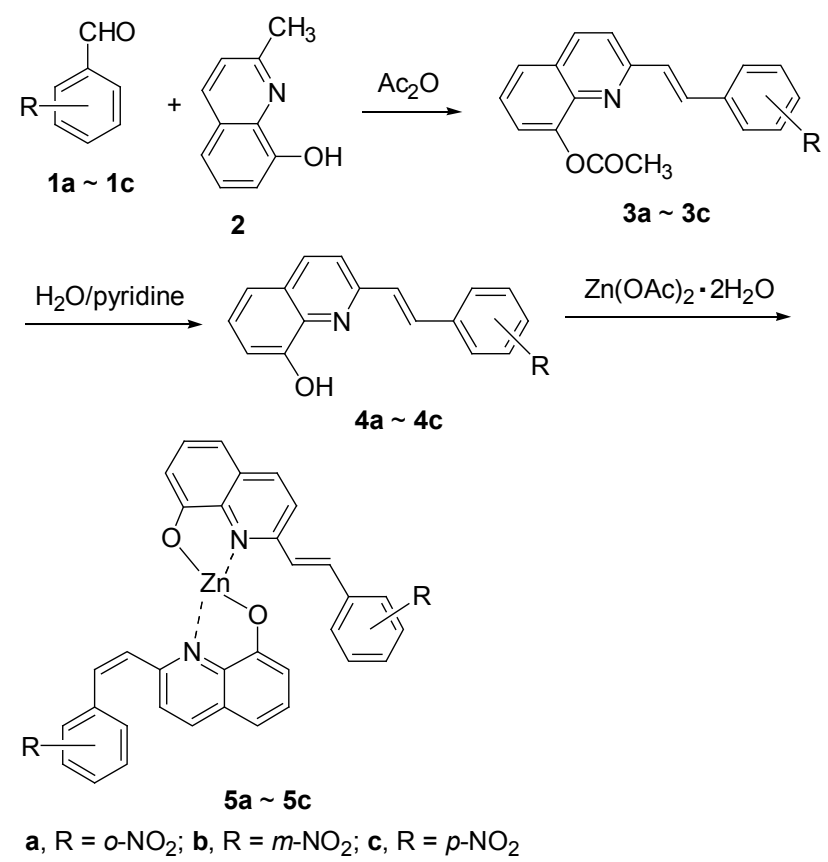

Scheme 1

\section{1 实验部分}

\section{1 仪器与试剂}

熔点采用 XT-4 双目显微镜熔点仪; UV 采用 TU-1901 双光束紫外可见分光光度计; IR 采用 NICOLET 380 傅里叶变换红外光谱仪; ${ }^{1} \mathrm{H}$ NMR 采用德 国-瑞士的 DRX-400 MHz 核磁共振仪, TMS 为外标, $\mathrm{CDCl}_{3}$ 为溶剂; 元素分析采用 Perkin-Elmer 2400 元素分 析仪; MS 采用 FINNIGAN Trace DSQ 气相色谱一质谱仪 (直接进样); 苂光仪型号为 Fluoromax-4; TGA (Thremo Gravimetric Analyzer)采用德国耐驰的 STA409P 热重分 析仪. 8-着基喹哪啶购自日本东京化成试剂公司, 邻、 间、对硝基苯甲醛购于上海晶纯试剂有限公司, 其它试 剂为市售分析纯.

\section{2 化合物 $3 a \sim 3 c$ 的合成}

称取 8-着基喹哪啶 $1.21 \mathrm{~g}(7.6 \mathrm{mmol}) 、$ 邻硝基苯甲 醛 $1.126 \mathrm{~g}(7.4 \mathrm{mmol})$ 和 $10 \mathrm{~mL}$ 乙酸酐, 加入到 $100 \mathrm{~mL}$ 的圆底烧瓶中. 搅拌加热, 温度 $145 \sim 148{ }^{\circ} \mathrm{C}$, 在氮气保 护下回流反应 $11 \mathrm{~h}$, 随着反应的进行, 反应物颜色逐渐 加深, 由黄色转为褐色, 反应溶液呈浑浊状, 有固体析 出. 固体用 $100 \mathrm{~mL}$ 水抽滤洗涤三次, 真空干燥得到 1.70 $\mathrm{g}$ 橙色粉末状固体化合物(E)-2-[2-(2-硝基苯基)乙烯 基]-8-乙酰氧基喹啉(3a), 产率 $62 \%$. m.p. $160 \sim 161{ }^{\circ} \mathrm{C}$; ${ }^{1} \mathrm{H}$ NMR $\left(\mathrm{CDCl}_{3}, 300 \mathrm{MHz}\right) \delta: 8.23(\mathrm{~d}, J=15.9 \mathrm{~Hz}, 1 \mathrm{H})$, 8.19 (d, $J=8.4 \mathrm{~Hz}, 1 \mathrm{H}), 7.99$ (d, $J=8.4 \mathrm{~Hz}, 1 \mathrm{H}), 7.90$ (d, $J=7.5 \mathrm{~Hz}, 1 \mathrm{H}), 7.68 \sim 7.60(\mathrm{~m}, 2 \mathrm{H}), 7.66(\mathrm{~d}, J=8.4 \mathrm{~Hz}$, $1 \mathrm{H}), 7.52(\mathrm{~d}, J=7.5 \mathrm{~Hz}, 1 \mathrm{H}), 7.50(\mathrm{~d}, J=7.5 \mathrm{~Hz}, 1 \mathrm{H})$, $7.48 \sim 7.40(\mathrm{~m}, 2 \mathrm{H}), 2.59$ (s, 3H); IR (KBr) $v: 3067,1723$, 1577, 1520, 1457, 1175, 1128, 970, 850, 765, $708 \mathrm{~cm}^{-1}$; ESI-MS $m / z: 335.9\left([\mathrm{M}+\mathrm{H}]^{+}\right)$. Ana1. calcd for $\mathrm{C}_{19} \mathrm{H}_{14} \mathrm{~N}_{2} \mathrm{O}_{4}$ : C 68.26, H 4.22, N 8.38; found C 68.10, H $4.42, \mathrm{~N} 8.46$.

(E)-2-[2-(3-硝基苯基)乙烯基]-8-乙酰氧基喹啉 $(3 \mathbf{b})$ 的制备方法与 3a 相同, 真空干燥得到黄色粉末状固体 化合物, 产率 61.4\%. m.p. $166 \sim 167{ }^{\circ} \mathrm{C} ;{ }^{1} \mathrm{H}$ NMR $\left(\mathrm{CDCl}_{3}, 400 \mathrm{MHz}\right) \delta: 8.45(\mathrm{~s}, 1 \mathrm{H}), 8.17$ (d, $J=8.8 \mathrm{~Hz}$, $1 \mathrm{H}), 8.16(\mathrm{~d}, J=8.8 \mathrm{~Hz}, 1 \mathrm{H}), 7.89(\mathrm{~d}, J=8.0 \mathrm{~Hz}, 1 \mathrm{H}), 7.70$ (d, $J=16.0 \mathrm{~Hz}, 1 \mathrm{H}), 7.69$ (d, $J=8.0 \mathrm{~Hz}, 1 \mathrm{H}), 7.64(\mathrm{~d}, J=$ $7.8 \mathrm{~Hz}, 1 \mathrm{H}), 7.57(\mathrm{t}, J=8.0 \mathrm{~Hz}, 1 \mathrm{H}), 7.51(\mathrm{t}, J=7.8 \mathrm{~Hz}$, $1 \mathrm{H}), 7.45(\mathrm{~d}, J=7.8 \mathrm{~Hz}, 1 \mathrm{H}), 7.44(\mathrm{~d}, J=15.6 \mathrm{~Hz}, 1 \mathrm{H})$, 2.59 (s, 3H), 2.59 (s, 3H); IR (KBr) $v: 3063,1733,1580$, 1527, 1453, 1180, 1117, 960, 870, 760, $703 \mathrm{~cm}^{-1}$; EI-MS $m / z: 335.43\left[(\mathrm{M}+1)^{+}\right], 246.66(\mathrm{M}-89)^{+}$. Ana1. calcd for $\mathrm{C}_{19} \mathrm{H}_{14} \mathrm{~N}_{2} \mathrm{O}_{4}$ : C 68.26, H 4.22, N 8.38; found C 68.35, H $4.32, \mathrm{~N} 8.49$.

(E)-2-[2-(4-硝基苯基)乙烯基]-8-乙酰氧基喹啉(3c) 的制备方法与 3a 相同, 真空干燥得到褐色粉末状固体 化合物, 产率 73.9\%. m.p. $163{ }^{\circ} \mathrm{C} ;{ }^{1} \mathrm{H}$ NMR $\left(\mathrm{CDCl}_{3}, 400\right.$ MHz) $\delta: 8.29 \sim 8.24(\mathrm{~m}, 2 \mathrm{H}), 8.19(\mathrm{~d}, J=8.4 \mathrm{~Hz}, 1 \mathrm{H})$, $7.79 \sim 7.73(\mathrm{~m}, 3 \mathrm{H}), 7.67(\mathrm{~d}, J=8.4 \mathrm{~Hz}, 1 \mathrm{H}), 7.53(\mathrm{~d}, J=$ $15.6 \mathrm{~Hz}, 1 \mathrm{H}), 7.46(\mathrm{~d}, J=7.2 \mathrm{~Hz}, 1 \mathrm{H}), 2.58$ (s, 3H); IR (KBr) v: 3061, 1723, 1567, 1527, 1448, 1177, 1123, 958, 867, 757, $709 \mathrm{~cm}^{-1}$; EI-MS m/z: $335.41\left[(\mathrm{M}+1)^{+}\right], 256.48$ $(\mathrm{M}-79)^{+}$. Ana1. calcd for $\mathrm{C}_{19} \mathrm{H}_{14} \mathrm{~N}_{2} \mathrm{O}_{4}$ : C 68.26, H 4.22, $\mathrm{N} 8.38$; found C 68.15, H 4.52, N 8.63.

\section{3 化合物 $4 a \sim 4 c$ 的合成}

称取 $1.00 \mathrm{~g}(3.0 \mathrm{mmol})$ 化合物 $3 \mathbf{a}, 10 \mathrm{~mL}$ 吡定加入到 $100 \mathrm{~mL}$ 圆底烧瓶中, 搅拌加热, 反应温度维持在 132 $135{ }^{\circ} \mathrm{C} .30 \mathrm{~min}$ 后, 加入 $5 \mathrm{~mL}$ 水到反应溶液中, 加入瞬 间，反应溶液有浑浊现象，有固体产生. 继续回流 $3 \mathrm{~h}$ 后, 停止反应, 加入冷水 $20 \mathrm{~mL}$. 放置冷却析出固体, $100 \mathrm{~mL}$ 水抽滤洗涤三次, 真空干燥得到淡黄色粉末化 合物(E)-2-[2-(2-硝基苯基)乙烯基]-8-羊基喹啉(4a) 0.74 g, 产率 $83.9 \%$. m.p. $170{ }^{\circ} \mathrm{C} ;{ }^{1} \mathrm{H}$ NMR $\left(\mathrm{CDCl}_{3}, 300 \mathrm{MHz}\right)$ $\delta: 8.34(\mathrm{~d}, J=8.7 \mathrm{~Hz}, 1 \mathrm{H}), 8.29(\mathrm{~d}, J=10.4 \mathrm{~Hz}, 1 \mathrm{H})$, $8.03 \sim 7.96(\mathrm{~m}, 2 \mathrm{H}), 7.83(\mathrm{~d}, J=8.7 \mathrm{~Hz}, 1 \mathrm{H}), 7.78(\mathrm{~d}, J=$ $8.1 \mathrm{~Hz}, 1 \mathrm{H}), 7.62(\mathrm{t}, J=8.1 \mathrm{~Hz}, 1 \mathrm{H}), 7.48(\mathrm{~d}, J=15.6 \mathrm{~Hz}$, 
1H), $7.46(\mathrm{~d}, J=15.6 \mathrm{~Hz}, 1 \mathrm{H}), 7.39$ (d, $J=8.4 \mathrm{~Hz}, 1 \mathrm{H})$, 7.12 (d, $J=7.2 \mathrm{~Hz}, 1 \mathrm{H}$ ); IR (KBr) v: 3466, 1610, 589, 1579, 874, $759 \mathrm{~cm}^{-1}$; ESI-MS m/z: $293.42\left[(\mathrm{M}+1)^{+}\right]$. Ana1. calcd for $\mathrm{C}_{17} \mathrm{H}_{12} \mathrm{~N}_{2} \mathrm{O}_{3}$ : C 69.86, H 4.14, N 9.58; found $\mathrm{C} 69.72, \mathrm{H} 4.22, \mathrm{~N} 9.63$.

(E)-2-[2-(3-硝基苯基)乙烯基]-8-羟基喹啉(4b)的制 备方法与 $4 \mathrm{a}$ 相同, 得到黄色粉末状固体化合物, 产率 70.6\%. m.p. $175{ }^{\circ} \mathrm{C} ;{ }^{1} \mathrm{H}$ NMR $\left(\mathrm{CDCl}_{3}, 400 \mathrm{MHz}\right) \delta: 8.49$ (t, $J=7.8 \mathrm{~Hz}, 1 \mathrm{H}), 8.18(\mathrm{dd}, J=10.4,1.4 \mathrm{~Hz}, 1 \mathrm{H}), 8.17$ (d, $J=8.4 \mathrm{~Hz}, 1 \mathrm{H}), 7.93(\mathrm{~d}, J=8.0 \mathrm{~Hz}, 1 \mathrm{H}), 7.77$ (d, $J=16.0$ $\mathrm{Hz}, 1 \mathrm{H}), 7.65(\mathrm{~d}, J=8.4 \mathrm{~Hz}, 1 \mathrm{H}), 7.59(\mathrm{t}, J=8.0 \mathrm{~Hz}, 1 \mathrm{H})$, $7.46(\mathrm{~d}, J=16 \mathrm{~Hz}, 1 \mathrm{H}), 7.44(\mathrm{t}, J=8.0 \mathrm{~Hz}, 1 \mathrm{H}), 7.33$ (dd, $J=8.4,1.2 \mathrm{~Hz}, 1 \mathrm{H}), 7.19$ (dd, $J=7.8,1.2 \mathrm{~Hz}, 1 \mathrm{H})$; IR (KBr) $v$ : 3462, 1641, 1583, 1569, 871, $769 \mathrm{~cm}^{-1}$; EI-MS $m / z: 293.47\left[(\mathrm{M}+1)^{+}\right], 246.66(\mathrm{M}-47)^{+}$. Anal. calcd for $\mathrm{C}_{17} \mathrm{H}_{12} \mathrm{~N}_{2} \mathrm{O}_{3}$ : C 69.86, H 4.14, N 9.58; found C 69.63, $\mathrm{H}$ $4.32, \mathrm{~N} 9.74$.

(E)-2-[2-(4-硝基苯基)乙烯基]-8-差基喹啉(4c) 的制 备方法与 $4 \mathbf{a}$ 相同, 得到橙黄色粉末状固体化合物, 产率 74.3\%. m.p. $200{ }^{\circ} \mathrm{C} ;{ }^{1} \mathrm{H}$ NMR $\left(\mathrm{CDCl}_{3}, 400 \mathrm{MHz}\right) \delta$ : $8.28 \sim 8.20(\mathrm{~m}, 2 \mathrm{H}), 8.18(\mathrm{~d}, J=8.4 \mathrm{~Hz}, 1 \mathrm{H}), 7.77(\mathrm{~d}, J=$ $15.6 \mathrm{~Hz}, 1 \mathrm{H}), 7.76 \sim 7.70(\mathrm{~m}, 2 \mathrm{H}), 7.66(\mathrm{~d}, J=7.8 \mathrm{~Hz}$, $1 \mathrm{H}), 7.48(\mathrm{~d}, J=15.6 \mathrm{~Hz}, 1 \mathrm{H}), 7.44(\mathrm{~d}, J=7.8 \mathrm{~Hz}, 1 \mathrm{H})$, 7.33 (d, $J=8.4 \mathrm{~Hz}, 1 \mathrm{H}), 7.20$ (d, $J=7.8 \mathrm{~Hz}, 1 \mathrm{H})$; IR (KBr) $v: 3460,1610,1577,1568,868,761 \mathrm{~cm}^{-1}$; EI-MS $\mathrm{m} / z$ : $293.52\left[(\mathrm{M}+1)^{+}\right], 246.64(\mathrm{M}-47)^{+}$. Anal. calcd for $\mathrm{C}_{17} \mathrm{H}_{12} \mathrm{~N}_{2} \mathrm{O}_{3}$ : C 69.86, H 4.14, N 9.58; found C 69.92, $\mathrm{H}$ $4.08, \mathrm{~N} 9.77$

\section{4 化合物 $5 a \sim 5 c$ 的合成}

称取 $0.146 \mathrm{~g}(0.5 \mathrm{mmol})$ 的化合物 $4 \mathbf{a}$, 吸取 $15 \mathrm{~mL}$ 甲 醇加入到 $100 \mathrm{~mL}$ 烧瓶, 摚拌溶解. 另称取醋酸锌固体 $0.12 \mathrm{~g}(0.55 \mathrm{mmol})$ 放入小烧杯中用 $15 \mathrm{~mL}$ 水溶解, 用滴 液漏斗缓慢滴入反应瓶中, 反应物呈浑浊黄色. 搅拌 24 $\mathrm{h}$ 后停止, 静置过滤, 用水和甲醇各 $100 \mathrm{~mL}$ 洗涤三次. 真空干燥得到黄色粉末状固体化合物 $(E)$-2-[2-(2-硝基苯 基)乙烯基]-8-羊基喹啉锌(5a) $0.130 \mathrm{~g}$, 产率 $80.2 \%$. ${ }^{1} \mathrm{H}$ NMR (DMSO- $\left.d_{6}, 400 \mathrm{MHz}\right) \delta: 8.62(\mathrm{~d}, J=16.8 \mathrm{~Hz}, 1 \mathrm{H})$, $8.43(\mathrm{~d}, J=8.4 \mathrm{~Hz}, 1 \mathrm{H}), 8.20$ (d, $J=8.0 \mathrm{~Hz}, 1 \mathrm{H}), 8.12$ (d, $J=8.4 \mathrm{~Hz}, 1 \mathrm{H}), 8.06$ (d, $J=8.0 \mathrm{~Hz}, 1 \mathrm{H}), 7.93$ (d, $J=16.4$ $\mathrm{Hz}, 1 \mathrm{H}), 7.83$ (t, $J=8.0 \mathrm{~Hz}, 1 \mathrm{H}), 7.61(\mathrm{~d}, J=8.4 \mathrm{~Hz}, 1 \mathrm{H})$, $7.44(\mathrm{t}, J=8.0 \mathrm{~Hz}, 1 \mathrm{H}), 7.02(\mathrm{~d}, J=8.0 \mathrm{~Hz}, 1 \mathrm{H}), 6.94(\mathrm{~d}$, $J=8.0 \mathrm{~Hz}, 1 \mathrm{H})$; IR (KBr) $v: 3043,1624,1560,838,748$, 560, $470 \mathrm{~cm}^{-1}$; FAB MS-MS m/z: $648(\mathrm{M}+\mathrm{H})^{+}$. Anal. calcd for $\mathrm{C}_{34} \mathrm{H}_{22} \mathrm{~N}_{4} \mathrm{O}_{6} \mathrm{Zn}$ : C 63.02, H 3.42, N 8.65; found C 63.44, H 3.08, N 8.40.
(E)-2-[2-(3-硝基苯基)乙烯基]-8-羊基喹啉锌 $(\mathbf{5 b}$ ) 的 制备方法与 5a 相同, 得到橙色粉末状固体化合物, 产率 81.7\%. ${ }^{1} \mathrm{H}$ NMR (DMSO- $d_{6}, 400 \mathrm{MHz}$ ) $\delta: 8.71$ (d, $J=16.4$ $\mathrm{Hz}, 1 \mathrm{H}), 8.33$ (d, $J=8.8 \mathrm{~Hz}, 1 \mathrm{H}), 8.20(\mathrm{~d}, J=8.0 \mathrm{~Hz}, 1 \mathrm{H})$, $8.14(\mathrm{~d}, J=8.4 \mathrm{~Hz}, 1 \mathrm{H}), 8.06(\mathrm{~d}, J=8.0 \mathrm{~Hz}, 1 \mathrm{H}), 7.93$ (d, $J=16.4 \mathrm{~Hz}, 1 \mathrm{H}), 7.83$ (t, $J=8.0 \mathrm{~Hz}, 1 \mathrm{H}), 7.61$ (d, $J=8.4$ $\mathrm{Hz}, 1 \mathrm{H}), 7.32$ (t, $J=8.0 \mathrm{~Hz}, 1 \mathrm{H}), 7.12(\mathrm{~d}, J=8.0 \mathrm{~Hz}, 1 \mathrm{H})$, 7.04 (d, $J=8.4 \mathrm{~Hz}, 1 \mathrm{H})$; IR (KBr) $v: 3043,1610,1543$, 830, 748, 554, $467 \mathrm{~cm}^{-1}$; FAB MS-MS m/z: $648(\mathrm{M}+\mathrm{H})^{+}$. Anal. calcd for $\mathrm{C}_{34} \mathrm{H}_{22} \mathrm{~N}_{4} \mathrm{O}_{6} \mathrm{Zn}$ : C 63.02, H 3.42, N 8.65; found $\mathrm{C} 63.24, \mathrm{H} 3.60, \mathrm{~N} 8.77$.

(E)-2-[2-(4-硝基苯基)乙烯基]-8-羟基喹啉锌 $(\mathbf{5 c})$ 的 制备方法与 5a 相同, 得到红褐色粉末状固体化合物, 产 率 87.1\%. ${ }^{1} \mathrm{H}$ NMR (DMSO- $\left.d_{6}, 400 \mathrm{MHz}\right) \delta: 8.33$ (d, $J=$ $8.8 \mathrm{~Hz}, 1 \mathrm{H}), 8.30(\mathrm{~d}, J=8.8 \mathrm{~Hz}, 1 \mathrm{H}), 8.28(\mathrm{~d}, J=16.8 \mathrm{~Hz}$, $1 \mathrm{H}), 8.27(\mathrm{~d}, J=8.0 \mathrm{~Hz}, 1 \mathrm{H}), 7.95(\mathrm{~d}, J=8.8 \mathrm{~Hz}, 2 \mathrm{H}), 7.80$ (d, $J=8.4 \mathrm{~Hz}, 1 \mathrm{H}), 7.70(\mathrm{~d}, J=16 \mathrm{~Hz}, 1 \mathrm{H}), 7.39$ (t, $J=7.6$ $\mathrm{Hz}, 1 \mathrm{H}), 7.37$ (d, $J=8.4 \mathrm{~Hz}, 1 \mathrm{H}), 7.09$ (d, $J=7.2 \mathrm{~Hz}, 1 \mathrm{H}$ ); IR (KBr) v: 3041, 1617, 1533, 842, 732, 542, $473 \mathrm{~cm}^{-1}$; FAB MS $m / z$ : $648(\mathrm{M}+\mathrm{H})^{+}$. Anal. calcd for $\mathrm{C}_{34} \mathrm{H}_{22} \mathrm{~N}_{4^{-}}$ $\mathrm{O}_{6} \mathrm{Zn}: \mathrm{C} 63.02, \mathrm{H} 3.42, \mathrm{~N} 8.65$; found C $63.48, \mathrm{H} 3.77, \mathrm{~N}$ 8.32 .

\section{2 结果与讨论}

\section{1 晶体结构的测定}

以乙酸乙酯一石油醚为溶剂，配制适量化合物 $3 \mathrm{a}$ 的 饱和溶液, 在室温下缓慢挥发, 约两周析出黄色单晶用 于结构测定，晶体的 $\mathrm{X}$ 射线数据是在室温下，由 Bruker SMART $1000 \mathrm{CCD}$ 衍射仪收集, 数据处理使用 $\mathrm{SAINT}^{+}$ 程序包, 对 1958 个 $I>2 \sigma(I)$ 的可观察点用直接法解析, 用全矩阵最小二乘法对 220 个参数进行修正, 对于非氢 原子采用各向异性热参数, 对于氢原子采用各向同性热 参数.

\section{2 晶体结构的描述}

在化合物 3a 的晶体结构中(表 1, 数据存于英国剑 桥数据中心, $\mathrm{CCDC}$ 号为 869913), C(1)和 $\mathrm{C}(2)$ 组成的平 面与 8-羟基喹啉环和硝基苯环的二面角分别为: $\mathrm{C}(1)-\mathrm{C}(2)-\mathrm{C}(6)-\mathrm{N}(5)=0.9(6)^{\circ}$ 和 $\mathrm{C}(2)-\mathrm{C}(1)-$ $\mathrm{C}(14)-\mathrm{C}(19)=1.1(7)^{\circ}$, 可知化合物 3a 的分子结构接近 理想平面, $\mathrm{C}(1)-\mathrm{C}(14)$ 为 $0.1459(2) \mathrm{nm}, \mathrm{C}(2)-\mathrm{C}(6)$ 为 0.1471(2) nm, 这两个 $\mathrm{C}-\mathrm{C}$ 单键比正常的 $\mathrm{C}-\mathrm{C}$ 单键键 长的平均值要小, 这是由于 $\mathrm{C}=\mathrm{C}$ 双键与喹啉环和硝基 苯环形成大的共扼体而使 $\mathrm{C}-\mathrm{C}$ 键长有明显缩短. 从图 1 可以看出化合物 $3 \mathbf{a}$ 的分子稳定构象为 $E$ 式结构, 由其 脱酯后得到的配体 $4 \mathrm{a}$ 也应为 $E$ 式结构, 这与通过 $4 \mathrm{a}$ 的 
氢谱烯烃邻位氢偶合常数 $15.6 \mathrm{~Hz}$, 判断的结果相一致. 配体 $4 \mathbf{b}$ 的 $\delta 7.77$ 和 7.46 处的两个氢的偶合常数为 16 $\mathrm{Hz}, 4 \mathrm{c}$ 的 $\delta 7.77$ 和 7.48 处的两个氢的偶合常数为 15.6 $\mathrm{Hz}$, 由此可知 $\mathbf{4 b}, \mathbf{4 c}$ 也为 $E$ 式结构.

表 1 化合物 3a 的 X 射线衍射数据

Table 1 X-ray diffraction data for compound 3a

\begin{tabular}{|c|c|}
\hline Empirical formula & $\mathrm{C}_{19} \mathrm{H}_{14} \mathrm{~N}_{2} \mathrm{O}_{4}$ \\
\hline Formula weight & 334.32 \\
\hline Temperature & $298(2) \mathrm{K}$ \\
\hline Wavelength & $0.71073 \AA$ \\
\hline Crystal system & Orthorhombic \\
\hline Space group & Pna2(1) \\
\hline Unit cell dimensions & $\begin{array}{l}a=2.58466(13) \mathrm{nm}, a=90^{\circ} \\
b=1.18451(6) \mathrm{nm}, \beta=90^{\circ} \\
c=1.05870(5) \mathrm{nm}, \gamma=90^{\circ}\end{array}$ \\
\hline$V$ & $324.13(3) \mathrm{nm}^{3}$ \\
\hline$Z$ & 8 \\
\hline Density (calculated) & $1.370 \mathrm{Mg} / \mathrm{m}^{3}$ \\
\hline Absorption coefficient & $0.098 \mathrm{~mm}^{-1}$ \\
\hline$F(000)$ & 1392 \\
\hline Crystal size & $0.47 \mathrm{~mm} \times 0.45 \mathrm{~mm} \times 0.26 \mathrm{~mm}$ \\
\hline$\theta$ range for data collection & $1.58^{\circ} \sim 27.08^{\circ}$ \\
\hline Limiting Indices & $\begin{array}{l}-29 \leqslant h \leqslant 33,-13 \leqslant k \leqslant 15 \\
-13 \leqslant l \leqslant 13\end{array}$ \\
\hline Reflections collected & 17451 \\
\hline Independent reflections & $3731[R($ int $)=0.0390]$ \\
\hline Completeness to $\theta=27.08^{\circ}$ & $99.1 \%$ \\
\hline Absorption correction & Semi-empirical from equivalents \\
\hline Max. and min. transmission & 0.9750 and 0.9555 \\
\hline Refinement method & Full-matrix least-squares on $F^{2}$ \\
\hline Data/restraints/parameters & $3731 / 7 / 453$ \\
\hline Goodness-of-fit on $F^{2}$ & 1.044 \\
\hline Final $R$ indices $[I>2 \sigma(I)]$ & $R_{1}=0.0578, w R_{2}=0.1434$ \\
\hline$R$ indices (all data) & $R_{1}=0.0831, w R_{2}=0.1714$ \\
\hline Largest diff. peak and hole & 0.0556 and $-0.0216 \mathrm{e} \cdot \mathrm{nm}^{-3}$ \\
\hline
\end{tabular}

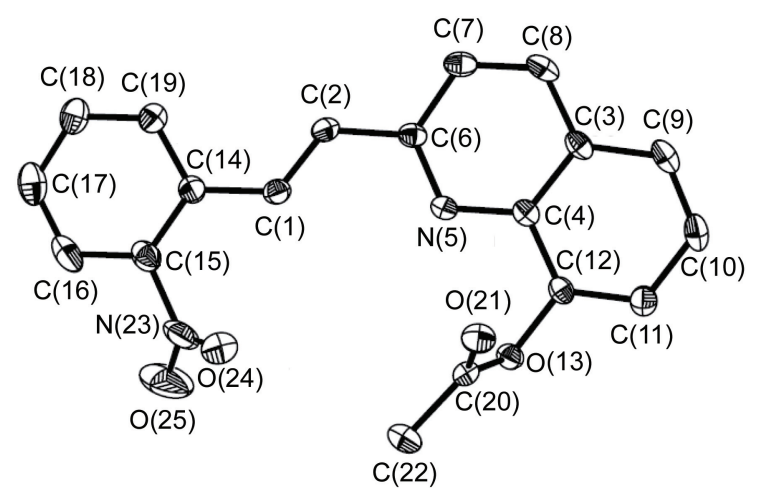

图 1 化合物 3a 的晶体结构

Figure 1 Molecular structure of $\mathbf{3 a}$

\section{3 配体与锌配位过程的紫外吸收图}

图 2 通过紫外滴定模拟了配体 $\mathbf{4 a}$ 与醋酸锌的配位
过程，随着醋酸锌的加入，在 $204 \mathrm{~nm}$ 处可以观察到明显 的减色效应，在 $230 \mathrm{~nm}$ 处出现了新的肩峰，而 $4 \mathbf{a}$ 在 342 $\mathrm{nm}$ 处的肩峰逐渐消失，生成的配合物 $\mathbf{5 a}$ 最大吸收波长 发生明显的红移，同时在 $426 \mathrm{~nm}$ 左右产生了新的吸收 峰. 图 3 的紫外滴定工作曲线表明: 当溶液中醋酸锌与 配体的物质的量的比大于 $1: 2$ 时, 紫外吸收光谱在 426 $\mathrm{nm}$ 处的吸光度不再发生变化，表明配位过程结束. 以 上现象模拟并验证了配合物的生成 ${ }^{[17]}$. 4b 和 4c 与醋酸 锌的配位过程与 $\mathbf{4 a}$ 类似, 数据见表 2 .

表 2 化合物 $4 \mathrm{a} \sim 4 \mathrm{c}$ 和 $5 \mathrm{a} \sim 5 \mathrm{c}$ 紫外吸收数据表

Table 2 The data of UV of compounds $4 a \sim 4 c$ and $5 a \sim 5 c$

\begin{tabular}{cccccc}
\hline 化合物 & $\lambda_{1} / \mathrm{nm}$ & $\lambda_{2} / \mathrm{nm}$ & $\lambda_{\max } / \mathrm{nm}$ & $\lambda_{3} / \mathrm{nm}$ & $\lambda_{4} / \mathrm{nm}$ \\
\hline $\mathbf{4 a}$ & 204 & - & 284 & 342 & - \\
$\mathbf{5 a}$ & 205 & 230 & 297 & - & 426 \\
$\mathbf{4 b}$ & 208 & - & 297 & 338 & - \\
$\mathbf{5 b}$ & 210 & 229 & 303 & - & 428 \\
$\mathbf{4 c}$ & 208 & - & 355 & 273 & - \\
$\mathbf{5 c}$ & 208 & 231 & 350 & - & 448 \\
\hline
\end{tabular}

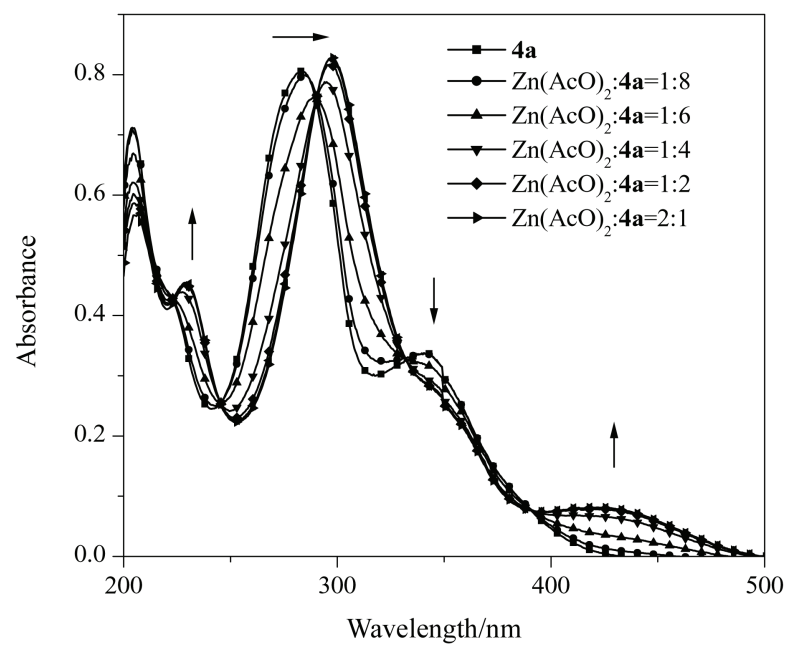

图 2 甲醇-水 $(V: V=9: 1)$ 溶液中化合物 $4 \mathbf{a}\left(6 \times 10^{-6} \mathrm{~mol}\right.$ • $\left.\mathrm{L}^{-1}\right)$ 与锌配位过程的紫外滴定图

Figure $2 \mathrm{UV}$-vis titrations of $4 \mathbf{a}\left(6 \times 10^{-6} \mathrm{~mol} \cdot \mathrm{L}^{-1}\right)$ with the addition of $\mathrm{Zn}^{2+}\left(2.4 \times 10^{-4} \mathrm{~mol} \cdot \mathrm{L}^{-1}\right)$ in $\mathrm{CH}_{3} \mathrm{OH}-\mathrm{H}_{2} \mathrm{O}(V: V=9: 1)$

\section{4 配体与锌配合物的荧光性质}

如图 4 所示, 配体 4a 4c 分别在 340, 305, $300 \mathrm{~nm}$ 的激发光激发下，其固体苂光发射波峰为 $516,556,536$ $\mathrm{nm}$, 而相应的锌配合物 $\mathbf{5 a} \sim \mathbf{5 c}$ 分别在 340, 305, $300 \mathrm{~nm}$ 的激发光激发下的固体苂光发射波峰为 596, 625, 592 $\mathrm{nm}$ : 配位以后，荧光发射发生了明显的红移，原因为： (1)金属配位后，金属与配体间的 $\pi$ 轨道反向偶合使得主 轨道的电子流动性增加, 金属离子与配体间形成离子 键; (2)配位后金属元素加入到环中加长了六元环的键 长, 通过抑制苯环电子的流动和增加刚性来形成一个非 


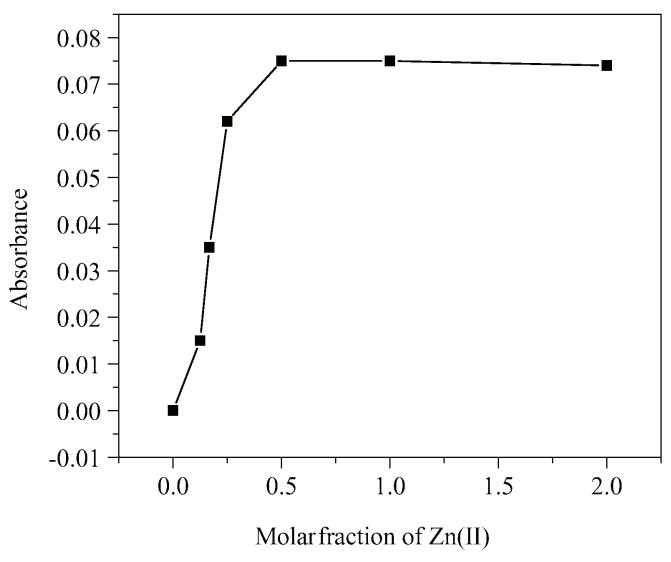

图 3 配体 $4 \mathrm{a}\left(6 \times 10^{-6}\right)$ 在波长为 $426 \mathrm{~nm}$ 的吸光度随着 $\mathrm{Zn}^{2+}$ $\left(2.4 \times 10^{-4} \mathrm{~mol} \cdot \mathrm{L}^{-1}\right)$ 加入的变化曲线

Figure 3 Job's plot of ligand $4 \mathrm{a}\left(6 \times 10^{-6}\right)$ and $\mathrm{Zn}^{2+}(2.4 \times$ $\left.10^{-4} \mathrm{~mol} \cdot \mathrm{L}^{-1}\right)$ at $426 \mathrm{~nm}$ against the molar fraction of $\mathrm{Zn}^{2+}$

辐射轨道以降低体系能量.

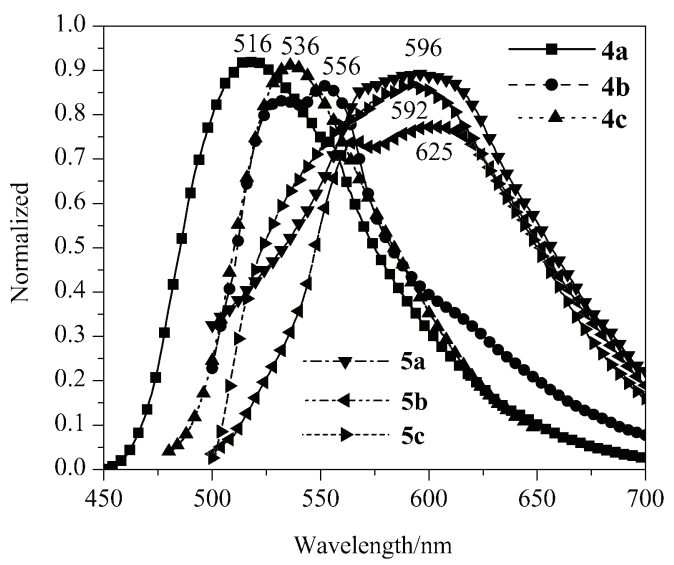

图 4 配体 $4 a \sim 4 c$ 和相应配合物 $5 a \sim 5 c$ 的固体苂光光谱

Figure 4 Fluorescence spectra of ligands $\mathbf{4 a} \sim \mathbf{4} \mathbf{c}$ and complexes $\mathbf{5 a} \sim \mathbf{5 c}$ in solid

如图 4所示, 配合物 $\mathbf{5 b}$ 的固体苂光发射波长红移最 明显，原因可能是硝基处于苯环间位时更有利于配合物 $\mathbf{5 b}$ 形成大的共轭平面，降低了体系的能量. 而配合物 $\mathbf{5 c}$ 的硝基处于苯环的对位, 由于空间阻碍作用, 不利于配 合物形成大的共轭平面，体系能量的降低不如 $5 \mathrm{~b}$ 明显.

如图 5 所示, $\mathbf{5 a} \sim \mathbf{5 c}$ 在 DMF 溶液中的 $\lambda_{\text {max }}$ 分别是 $562,536,618 \mathrm{~nm}$. 结果表明 $\mathbf{5 a} \sim \mathbf{5 c}$ 的固体荧光相对于 液体荧光发生了红移, 原因可能是固态时 $\mathbf{5 a}, \mathbf{5 b}$ 共轭程 度大, 分子间距离非常小, 相互之间作用力很明显, 易 生成激基缔合物或激基复合物, 导致激发时, 需要的能 量较小. 另外, 固态时, 避免了溶剂分子和化合物分子 间的相互作用, 电子跃迁更容易, 从而使固态时发射波 长红移. 而 $5 \mathrm{c}$ 固体荧光相对于液体荧光发生了蓝移, 原 因可能是 $5 \mathrm{c}$ 的硝基处于苯环的对位，由于空间阻碍的 作用, 其固态时共轭程度较低, 分子间距离较大, 相互
之间作用力不明显，导致激发时，相对于溶液中需要的 能量较大. 以上实验结果充分说明通过改变硝基的位置 可以调控其发光波长 ${ }^{[17 ~ 19]}$.

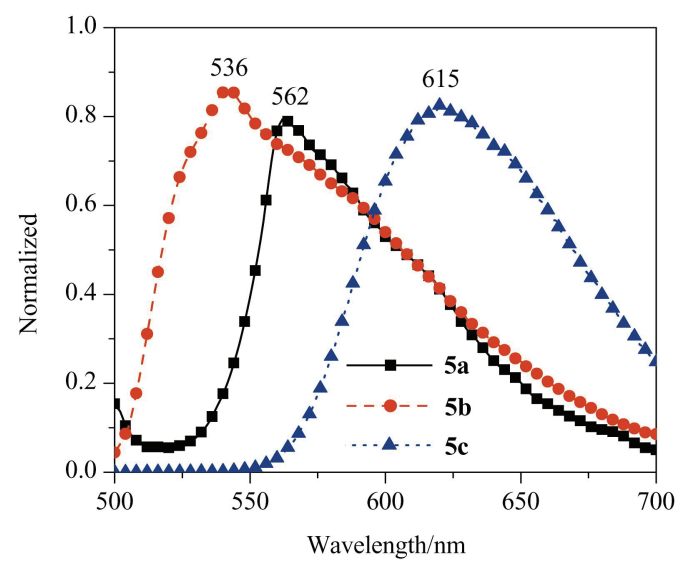

图 5 配合物 $5 \mathrm{a} \sim \mathbf{5} \mathrm{c}$ 在 DMF 溶液中的苂光光谱

Figure 5 Fluorescence spectra of compound $\mathbf{5 a} \sim \mathbf{5 c}$ in DMF

以硫酸奎宁的荧光量子产率 0.55 为标准 ${ }^{[20]}$, 测量 了 5a 5c 在 DMF 溶液中的荧光量子产率. 配制适当浓 度的配合物溶液和硫酸奎宁溶液, 使两者的吸光度 $A$ 相 近且不大于 0.05 , 扫描吸收光谱和苂光光谱, 分别得到 标准与样品的吸光度值 $\left(A_{\mathrm{R}}, A_{\mathrm{U}}\right)$ 和积分荧光强度 $\left(F_{\mathrm{R}}\right.$, $\left.F_{\mathrm{U}}\right)$. 再根据如下公式 ${ }^{[21]}$ 计算苂光量子产率 $\Phi_{\mathrm{fU}}$.

$$
\Phi_{\mathrm{fU}}=n_{\mathrm{U}}^{2} F_{\mathrm{U}} A_{\mathrm{R}} \Phi_{\mathrm{fR}} /\left(n_{\mathrm{R}}^{2} F_{\mathrm{R}} A_{\mathrm{U}}\right)
$$

5a $\sim 5 \mathbf{c}$ 的荧光量子产率计算结果分别为: 0.16 , $0.24,0.18$, 其中 $\mathbf{5 b}$ 的苂光量子产率最高，原因可能是硝 基处于间位时，更利于配合物 $\mathbf{5 b}$ 形成刚性平面.

\section{5 锌配合物的 TGA 图}

我们对化合物 5a $\sim \mathbf{5 c}$ 做了 $30 \sim 1000{ }^{\circ} \mathrm{C}$ 之间热重 分析. 升温速率为 $10{ }^{\circ} \mathrm{C} / \mathrm{min}$, 测量温度范围为室温 $1000{ }^{\circ} \mathrm{C}$, 所得的 TGA 曲线如图 6 所示. 从图中可以看

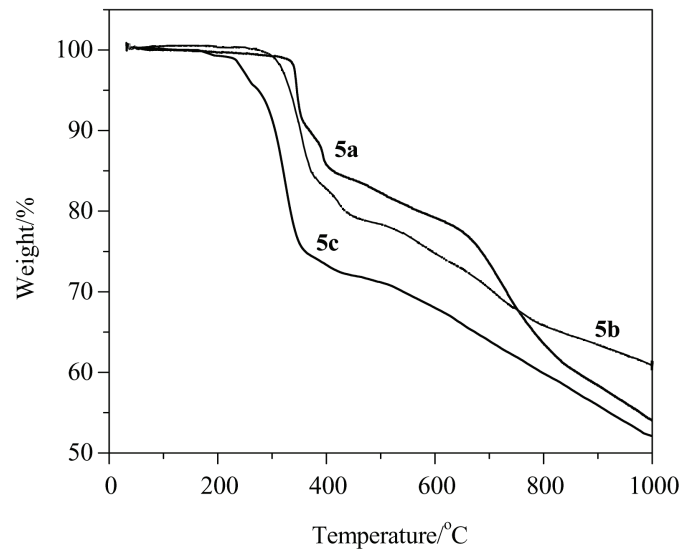

图 6 化合物 $5 \mathrm{a} \sim 5 \mathrm{c}$ 的 $\mathrm{TGA}$ 曲线图

Figure 6 TGA of compounds $\mathbf{5 a} \sim \mathbf{5 c}$ 
到化合物 5a～5c 在第一阶段的失重范围分别在 316 $669,280 \sim 671,225 \sim 522{ }^{\circ} \mathrm{C}$, 分别分解了 $24.4 \%, 28 \%$, $30 \%$. 此系配合物 5a $\sim 5 \mathbf{c}$ 开始逐渐分解失去配体，表明 锌配合物热稳定性好, 有利于真空蒸镀.

\section{References}

[1] Aziz, H.; Popovic, Z.; Hu, N.; Hor, A.; Xu, G. Science 1999, 283, 1900

[2] Friend, R. H.; Gymer, R. W. Nature 1999, 397, 121.

[3] Chen, Z.-B.; Liu, Y.-F. Chemistry 2001, 6, 345 (in Chinese). (陈兆涁, 刘玉芳, 化学通报, 2001, 6, 345.)

[4] Tang, C.-W.; Slyke, S. A. Appl. Phys. Lett. 1987, 53, 913.

[5] Mishra, A.; Nayak, P. K.; Periasamy, N. Tetrahedron Lett. 2004, 45,6265 .

[6] Xie, J.; Ning, Z.; Tian, H. Tetrahedron Lett. 2005, 46, 8559.

[7] Radek, P.; Pavel A, J. Org. lett. 2003, 5, 2769

[8] Huo, Y.-P.; Zhu, S.-Z. Chin. J. Chem. 2010, 28, 1389.

[9] Huo, Y.-P.; Zhu, S.-Z. Tetrahedron 2010, 66, 8635.

[10] Au, V. K.-M.; Wong, K. M.-C.; Tsang, D. P.-K.; Chan, M.-Y.; Zhu, N.-Y.; Yam, V. W.-W. J. Am. Chem. Soc. 2010, 132, 14273

[11] Lee, T. K.-M.; Zhu, N.-Y.; Yam, V. W.-W. J. Am. Chem. Soc. 2010, 132, 17646

[12] Zhou, Y.-D.; Zeng, H.-P.; Jing, H.-L.; Yuan, G.-Z.; Ouyang, X.-H. Chin. J. Org. Chem. 2006, 26, 1225 (in Chinese).
(周亚东, 曾和平, 靖慧莲, 袁国赞, 欧阳新华, 有机化学, 2006, 26, 1225.)

[13] Ouyang, X.-H.; Zeng, H.-P. Chin. J. Org. Chem. 2005, 25, 1410 (in Chinese). (欧阳新华, 曾和平, 有机化学, 2005, 25, 1410.)

[14] Li, D.-S.; Wang, Y.-Y.; Liu, P.; Luan, X. J.; Zhou, C.-H.; Shi, Q.-Z. Acta Chim. Sinica 2005, 63, 1633 (in Chinese). (李东升, 王尧宇, 刘萍, 栾新军, 周彩华, 史启祯, 化学学报, 2005, 63, 1633.)

[15] Xie, J.; Fan, L.; Su, J.; Tian, H. Dyes Pigm. 2003, 59, 153

[16] (a) Praveen, L.; Reddy, M. L. P.; Luxmi Varma, R. Tetrahedron Lett. 2010, 51(50), 6626.

(b) Chang, F.-S.; Chen, W.-C.; Wang, C.-H.; Tzeng, C.-C.; Chen, Y.-L. Bioorg. Med. Chem. 2010, 18(1), 124.

[17] Wang, T.-T.; Zeng, G.-C.; Zeng, H.-P.; Liu, P.-Y.; Wang, R.-X.; Zhang, Z.-J.; Xiong, Y.-L. Tetrahedron 2009, 65, 6325.

[18] Zeng, H.-P.; Wang, G.-R.; Zeng, G.-C.; Li, J. Dyes Pigm. 2009, 83, 155.

[19] (a) Zeng, F.-H.; Ni, J.; Wang, Q.-G.; Ding, Y.-B.; Ng, S.-W.; Zhu, W.-H.; Xie, Y.-S. Cryst. Growth Des. 2010, 10, 1611.

(b)Wei, K.-J.; Xie, Y.-S.; Ni, J.; Zhang, M.; Liu, Q.-L. Cryst. Growth Des. 2006, 6, 1341.

[20] Meech, S. R. J. Photochem. 1983, 23, 193.

[21] Posokhov, Y.; Kus, M.; Biner, H.; Gümüs, M. K. J. Photochem. Photobio1., A 2004, 161, 247.

(Zhao, C.; Fan, Y.) 